\title{
Evolução e qualidade de vida em pós-operatório de cirurgia vascular
}

\author{
Evolution and quality of life in postoperative vascular surgery \\ Evolución y calidad de vida en cirurgía vascular posoperatoria
}

Recebido: 19/01/2021 | Revisado: 26/01/2021 | Aceito: 05/02/2021 | Publicado: 11/02/2021

João Pedro Sousa Mendes

ORCID: https://orcid.org/0000-0002-0721-2845

Universidade Federal do Delta do Parnaíba, Brasil

E-mail: mendespedro00@gmail.com

Adrielly Cristhine Gonçalves Araújo

ORCID: https://orcid.org/0000-0002-2925-2231

Universidade Federal do Delta do Parnaíba, Brasil E-mail: adriellygoncalves@ufpi.edu.br

Cláudio Vinícius Barroso Queirós de Lima

ORCID: https://orcid.org/0000-0002-4047-8637

Universidade Federal do Delta do Parnaíba, Brasil

E-mail: cld.vinicius99@gmail.com

Igor dos Santos Cavalcante

ORCID: https://orcid.org/0000-0003-2907-9026

Universidade Federal do Delta do Parnaíba, Brasil

E-mail: igorsc@live.com

João Victor Carvalho Barbosa

ORCID: https://orcid.org/0000-0001-7790-0312

Universidade Federal do Delta do Parnaíba, Brasil

E-mail: joaovictorcarvalhob@gmail.com

Priscylla Frazão Rodrigues

ORCID: https://orcid.org/0000-0001-7580-516X

Universidade Federal do Delta do Parnaíba, Brasil

E-mail: priscyllafrazao1995@gmail.com

Olívio Joaquim Fonseca Neto

ORCID: https://orcid.org/0000-0002-2695-0077

Universidade Federal do Delta do Parnaíba, Brasil

E-mail: oliviofonsecaneto@ hotmail.com

Andressa Carvalho Pereira

ORCID: https://orcid.org/0000-0001-7721-0661

Universidade Federal do Delta do Parnaíba, Brasil

E-mail: andressa_carvalho@ufpi.edu.br

Brenda Larissa Andrade Viana

ORCID: https://orcid.org/0000-0003-2285-4309

Universidade Federal do Delta do Parnaíba, Brasil

E-mail: brendalarissa.av@ gmail.com

Isabella Pires Gomes Mendes

ORCID: https://orcid.org/0000-0003-3486-7908

Universidade Federal do Delta do Parnaíba, Brasil

E-mail: isabellapiresmendes@gmail.com

Juliano Luiz de Sousa

ORCID: https://orcid.org/0000-0003-0567-2167

Universidade Federal do Delta do Parnaíba, Brasil

E-mail: julianoluizdesouza@ufpi.edu.br

Yasser da Silveira Krüguer

ORCID: https://orcid.org/0000-0002-6163-7553

Universidade Federal do Delta do Parnaíba, Brasil

E-mail: yasser.kruguer@gmail.com

Elias de Carvalho Magalhães Neto

ORCID: https://orcid.org/0000-0002-7494-7147

Universidade Federal do Delta do Parnaíba, Brasil E-mail: elias.nt@hotmail.com

Érica de Araújo Silva Mendes

ORCID: https://orcid.org/0000-0001-5003-037X

Universidade Federal do Delta do Parnaíba, Brasil

E-mail: ericasilva.ma@gmail.com 


\begin{abstract}
Resumo
Este estudo tem como objetivo analisar e avaliar a qualidade de vida na recuperação de pacientes no período de pósoperatório em cirurgias vasculares. Métodos: trata-se de uma revisão de literatura integrativa, a qual se realizou uma pesquisa bibliográfica nas seguintes bases de dados, selecionadas através do metabuscador do Portal Periódico CAPES: Scientific Electronic Library Online (SciELO), Medical Literature Analysis and Retrievel System Online (Medline) e Literatura Latino-Americana e do Caribe em Ciências da Saúde (LILACS), utilizando os descritores "Cirurgia vascular", "Pós-operatório" e "Qualidade de vida", que foram pesquisados nos Descritores em Ciências da Saúde (DECs). Após a leitura analítica, apenas 14 artigos atenderam aos critérios de inclusão e foram utilizados para compor a amostra final deste estudo. Foram utilizados os artigos que estivessem enquadrados como estudos com seres humanos e publicados entre 2010 e 2020 . Resultados: a técnica cirúrgica tem grande influência na evolução do quadro clínico do paciente. Novas técnicas surgem com o objetivo de ser menos invasivas e proporcionarem uma melhor recuperação. Porém, algumas patologias, por serem graves, ainda apresentam uma alta taxa de mortalidade e complicações. Conclusão: Pacientes acometidos por doenças vasculares necessitam de um cuidado especial no intuito de evitar complicações no pós-operatório e garantir uma boa qualidade de vida. Observou-se que além da gravidade da doença, a avaliação da realidade que o paciente está inserido tem importância considerável na qualidade de vida, uma vez que os fatores biopsicossociais impactam de forma significativa no processo de adoecimento.
\end{abstract}

Palavras-chave: Cirurgia vascular; Pós-operatório; Qualidade de vida.

\begin{abstract}
This study aims to analyze and evaluate the quality of life in the recovery of patients in the postoperative period in vascular surgeries. Methods: this is an integrative literature review, which carried out a bibliographic search in the following databases, selected through the CAPES Periodic Portal metabolisador: Scientific Electronic Library Online (SciELO), Medical Literature Analysis and Retrievel System Online ( Medline) and Latin American and Caribbean Literature in Health Sciences (LILACS), using the descriptors "Vascular surgery", "Postoperative" and "Quality of life", which were searched in the Health Sciences Descriptors (DECs) . After analytical reading, only 14 articles met the inclusion criteria and were used to compose the final sample of this study. Articles that were classified as studies with human beings and published between 2010 and 2020 were used. Results: the surgical technique has a great influence on the evolution of the patient's clinical condition. New techniques appear with the objective of being less invasive and providing a better recovery. However, some pathologies, being serious, still have a high rate of mortality and complications. Conclusion: Patients with vascular diseases need special care in order to avoid complications in the postoperative period and ensure a good quality of life. It was observed that in addition to the severity of the disease, the assessment of the reality that the patient is inserted has considerable importance in the quality of life, since the biopsychosocial factors have a significant impact on the illness process.
\end{abstract}

Keywords: Vascular surgery; Postoperative; Quality of life.

\title{
Resumen
}

Este estudio tiene como objetivo analizar y evaluar la calidad de vida en la recuperación de pacientes en el pos-cirugía en cirugías vasculares. Métodos: se trata de una revisión integradora de la literatura, en la que se realizó una búsqueda bibliográfica en las siguientes bases de datos, seleccionadas a través del Portal Periódico CAPES metabolizador: Scientific Electronic Library Online (SciELO), Medical Literature Analysis and Retrievel System Online (Medline) y Latinoamericanas y Literatura del Caribe en Ciencias de la Salud (LILACS), utilizando los descriptores "Cirugía vascular", "Postoperatorio" y "Calidad de vida", que fueron buscados en los Descriptores de Ciencias de la Salud (DEC). Después de la lectura analítica, solo 14 artículos cumplieron con los criterios de inclusión y fueron utilizados para componer la muestra final de este estudio. Se utilizaron artículos que fueron clasificados como estudios con seres humanos y publicados entre 2010 y 2020. Resultados: la técnica quirúrgica tiene una gran influencia en la evolución de la condición clínica del paciente. Aparecen nuevas técnicas con el objetivo de ser menos invasivas y proporcionar una mejor recuperación. Sin embargo, algunas patologías, al ser graves, aún tienen una alta tasa de mortalidad y complicaciones. Conclusión: Los pacientes con enfermedades vasculares necesitan cuidados especiales para evitar complicaciones en el postoperatorio y asegurar una buena calidad de vida. Se observó que además de la gravedad de la enfermedad, la valoración de la realidad en que se inserta el paciente tiene una importancia considerable en la calidad de vida, ya que los factores biopsicosociales tienen un impacto significativo en el proceso de la enfermedad.

Palabras clave: Cirugía vascular; Postoperatorio; Calidad de vida.

\section{Introdução}

A qualidade de vida está correlacionada à concepção do indivíduo com sua situação física e emocional, também atrelada aos seus sentimentos, suas experiências e vivências atuais. No contexto hospitalar, procedimentos invasivos e complexos, que demandam um pós-operatório mais cauteloso, podem ser um gerador de estresse e preocupações, afetando os estados emocionais dos pacientes (Giaretta, Vigueras, Ruschel, \& Souza, 2018). 
São várias as patologias vasculares que necessitam de intervenções cirúrgicas para o seu tratamento, dentre as mais comuns têm-se a insuficiência venosa crônica, doença arterial obstrutiva periférica (DAOP), aneurisma da aorta abdominal (AAA), revascularização de membros inferiores e a dissecção de aorta. Esses procedimentos caracterizam-se por apresentarem um risco elevado para complicações que, em alguns casos, podem causar a morte. Por esse motivo, após um procedimento cirúrgico, o período de recuperação pós-operatória é essencial para que o paciente possa restabelecer sua saúde e poder voltar às suas atividades cotidianas. De fato, esse processo é complexo e multidimensional, tornando necessária uma ampla visão clínica, objetivando restabelecer as capacidades e a homeostase após o procedimento cirúrgico. Além disso, o processo de adoecimento juntamente ao pós operatório afetam física e mentalmente os pacientes, o que reflete na qualidade de vida desses indivíduos, demonstrando, ainda mais, a importância do acompanhamento por todo o processo no pré, peri e pós-operatório.

A aterosclerose, um dos motivos de revascularização de membros e de dissecção de aorta, representa um terço das causas de óbitos no Brasil, sendo o principal fator causador das doenças arteriais obstrutivas coronarianas, cerebrovasculares e arteriais periféricas. Cerca de $60 \%$ dos portadores de DAOP também possuem algum acometimento coronariano e cerebrovascular (Pereira, Oliveira, Carvalho, \& Bellen, 2016). Os procedimentos cirúrgicos, dessa forma, por apresentarem riscos elevados de complicações durante e no pós-operatório, requerem atenção por parte da equipe médica para que possa agir em situações de prognóstico ruim. Algumas complicações possíveis provenientes de cirurgias vasculares são aquelas que comprometem o sistema cardiovascular, dentre elas, observa-se elevados índices de infarto agudo do miocárdio, insuficiência cardíaca, arritmias e acidente vascular cerebral (Smeili \& Lotufo, 2015).

Pacientes submetidos a cirurgias vasculares necessitam de um determinado cuidado no pós-operatório. Por serem procedimentos, em sua maioria, complexos e com repercussões orgânicas significativas, o pós-operatório requer cuidados intensivos com o intuito de estabelecer uma recuperação adequada aos pacientes e evitar complicações.

Assim, a devida recuperação pós-operatória é essencial para evitar desfechos e consequências adversos em longo prazo. É de grande importância que a equipe de saúde acompanhe o paciente nesse processo de recuperação e esteja preparada para identificar de forma precoce e precisa esses pacientes a fim de facilitar intervenções oportunas de prevenção de tais efeitos adversos (Ferraz et al. 2018). À vista disso, este estudo tem como objetivo analisar e avaliar a qualidade de vida na recuperação de pacientes no período de pós-operatório em cirurgias vasculares.

\section{Metodologia}

Trata-se de uma revisão de literatura integrativa, cuja abordagem adotada foi qualitativa, visando avaliar a qualidade de vida na recuperação pós-operatória de cirurgias vasculares, analisando diferentes procedimentos cirúrgicos e como algumas técnicas influenciaram na evolução do quadro do paciente. Essa pesquisa levou em consideração os fundamentos de uma revisão integrativade literatura, os quais consistem em: (1) elaboração da pergunta central da pesquisa; (2) investigação na literatura; (3) seleção de estudos; (4) avaliação metodológica; (5) síntese dos dados por meio de análise descritiva; (6) avaliação da qualidade das evidências e (7) escrita e publicação dos resultados.

A pesquisa bibliográfica foi realizada nas seguintes bases de dados, selecionadas através do metabuscador do Portal Periódico CAPES: Scientific Electronic Library Online (SciELO), Medical Literature Analysis and Retrievel System Online (Medline) e Literatura Latino-Americana e do Caribe em Ciências da Saúde (LILACS), utilizando os descritores "Cirurgia vascular", "Pós-operatório" e "Qualidade de vida", que foram pesquisados nos Descritores em Ciências da Saúde (DECs). Para aprimoramento da busca, foi utilizado o operador booleano "AND" e selecionados artigos de relevância para o tema.

A seleção dos estudos foi realizada de forma independente por dois pesquisadores com experiência em revisões, a partir da analise dos títulos, resumos e textos completos das publicações. As divergências foram resolvidas por consenso e, na sua impossibilidade, por um terceiro pesquisador com experiência no tema. Todos os artigos passaram pelo crivo metodológico 
dos autores, sendo que os estudos com abordagem distinta da proposta inicial foram excluídos. Os critérios de inclusão foram: artigos científicos disponibilizados de forma gratuita e integral, nos idiomas inglês, português ou espanhol, que fossem pesquisas em seres humanos, cujo tema apresentasse foco nos resultados de algum tipo de cirurgia vascular, analisando suas complicações, o prognóstico e/ou o pós-operatório dos pacientes, publicados entre os anos 2010 e 2020. Os critérios de exclusão foram: materiais que não fossem artigos científicos, duplicidade de registros em mais de uma base de dados (excluídos automaticamente durante a busca), publicações anteriores ao ano de 2010, a fim de evitar a adesão de estudos desatualizados, e trabalhos que não tivessem relação com o tema selecionado.

Ao pesquisar na base de dados SciELO os três descritores associados encontrou-se 9 artigos, em seguida, utilizando-se os pares "Cirurgia vascular" e "Pós-operatório" a busca encontrou 194 artigos, já com o par "Cirurgia vascular" e "Qualidade de vida" não foi encontrado nenhum artigo na busca. Por fim, o par "Pós-operatório" e "Qualidade de vida" não foi utilizado na pesquisa para evitar tangenciamento ao tema.

$\mathrm{Na}$ base Medline, a combinação do trio associado não obteve resultados, o par "Cirurgia vascular" e "Qualidade de vida" encontrou 2 artigos e utilizando-se o par "Cirurgia Vascular" e "Pós-operatório" encontrou-se 12 artigos. Seguindo o padrão, o par "Pós-operatório" e "Qualidade de vida" não foi pesquisado a fim de evitar o tangeciamento ao tema.

Já na base LILACS, a busca pelos três termos em conjunto resultou em 9 artigos, o par "Cirurgia vascular" e "Qualidade de vida" resultou em 45 artigos, com o segundo par "Cirurgia Vascular" e "Pós-operatório" a busca encontrou 417 artigos. Novamente, o par "Pós-operatório" e "Qualidade de vida" foi descartado para evitar o tangeciamento ao tema.

Dos 688 artigos encontrados na busca inicial, 356 artigos foram excluídos inicialmente por não se adequarem ao recorte temporal, publicados antes do ano de 2010. Em seguida, foi realizada a leitura do título dos artigos e aqueles que não se enquadravam no tema estudado foram descartados, o que levou a exclusão de mais 151 documentos por esse motivo. Assim, um total de 181 artigos sobrou para a leitura dos resumos, levando a posterior eliminação de 159 artigos por não se adequarem ao tema do estudo. Dos 22 artigos restantes, realizou-se a leitura completa destes e exclui-se mais 8 por não serem úteis ao objetivo do estudo. Assim sendo, foram considerados válidos e incluídos nessa revisão 14 artigos para a realização da revisão de literatura. As etapas da pesquisa encontram-se na Figura 1. Com o intuito de proporcionar maior visibilidade e objetividade dos dados obtidos nos quartoze artigos selecionados, foi construída uma tabela apresentada abaixo (Tabela 1). 
Figura 1. Fluxograma detalhando cada etapa do processo de seleção de artigos para a revisão.
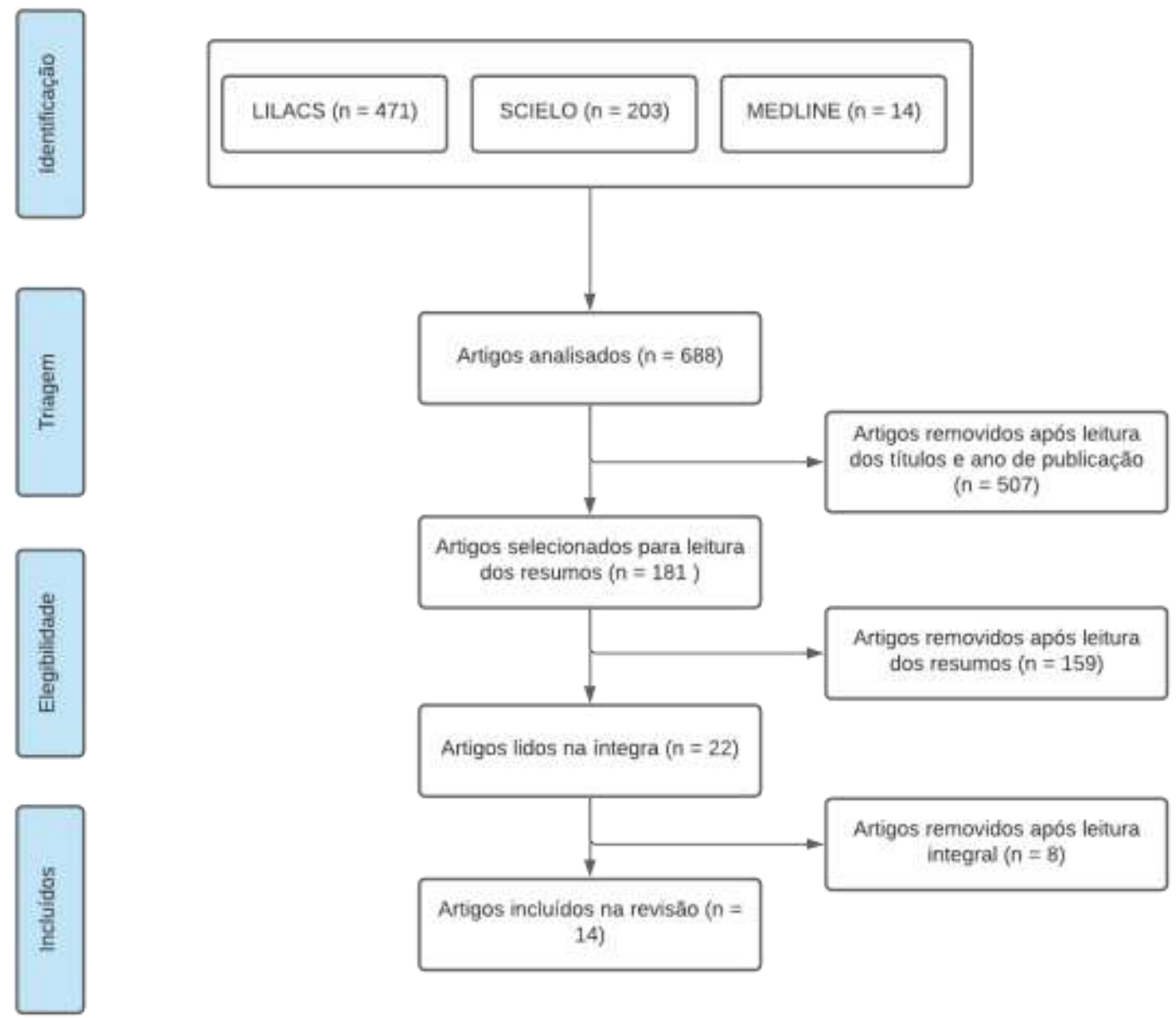

Fonte: Mendes et al. (2021).

\section{Resultados e Discussão}

Tabela 1. Artigos selecionados para composição do estudo, abordando a revista, ano de publicação e objetivos.

\begin{tabular}{|c|c|c|c|}
\hline TÍTULO & ANO & PERIÓDICO & OBJETIVO \\
\hline $\begin{array}{l}\text { Acute myocardial infarctation in } \\
\text { patients with critical ischemia } \\
\text { underwent lower limb } \\
\text { revascularization }\end{array}$ & 2013 & $\begin{array}{l}\text { Jornal Vascular } \\
\text { Brasileiro }\end{array}$ & $\begin{array}{l}\text { Avaliar incidência do infarto agudo do miocárdio no } \\
\text { pós-operatório imediato de pacientes submetidos à } \\
\text { revascularização de membros inferiores devido a } \\
\text { DAOP }\end{array}$ \\
\hline $\begin{array}{l}\text { Avaliação da qualidade de vida em } \\
\text { pacientes portadores de varizes de } \\
\text { membros inferiores submetidos a } \\
\text { tratamento cirúrgico }\end{array}$ & 2020 & $\begin{array}{l}\text { Jornal Vascular } \\
\text { Brasileiro }\end{array}$ & $\begin{array}{c}\text { Avaliar a qualidade de vida antes e após o tratamento } \\
\text { cirúrgico de pacientes portadores de varizes dos } \\
\text { membros inferiores }\end{array}$ \\
\hline $\begin{array}{l}\text { CHIVA to spare the small and great } \\
\text { saphenous veins after wrong-site } \\
\text { surgery on a normal saphenous } \\
\text { vein: a case report }\end{array}$ & 2019 & $\begin{array}{l}\text { Jornal Vascular } \\
\text { Brasileiro }\end{array}$ & $\begin{array}{l}\text { Apresentar o caso de uma paciente que foi submetida à } \\
\text { técnica cirúrgica Cure Conservatrice et } \\
\text { Hemodynamique de l'Insufficience Veineuse en } \\
\text { Ambulatoire (CHIVA) em duas safenas para poupá-las }\end{array}$ \\
\hline $\begin{array}{c}\text { Cardiac complications in vascular } \\
\text { surgery }\end{array}$ & 2016 & $\begin{array}{c}\text { Jornal Vascular } \\
\text { Brasileiro }\end{array}$ & $\begin{array}{l}\text { Determinar a prevalência da doença coronariana em } \\
\text { pacientes submetidos a cirurgia vascular eletiva de }\end{array}$ \\
\hline
\end{tabular}




\begin{tabular}{|c|c|c|c|}
\hline & & & $\begin{array}{c}\text { grande porte e sua relação com as complicações } \\
\text { cardiológicas pós-operatórias }\end{array}$ \\
\hline $\begin{array}{l}\text { Hérnias incisionais no pós- } \\
\text { operatório de correção de } \\
\text { aneurisma de aorta abdominal }\end{array}$ & 2012 & $\begin{array}{l}\text { Jornal Vascular } \\
\text { Brasileiro }\end{array}$ & $\begin{array}{l}\text { Apresentar a incidência de hérnia incisional em um } \\
\text { grupo de pacientes acompanhados no pós-operatório da } \\
\text { correção aberta de aneurisma de aorta abdominal }\end{array}$ \\
\hline $\begin{array}{l}\text { Incidência e preditores de } \\
\text { complicações cardiovasculares e } \\
\text { óbito após cirurgia vascular arterial }\end{array}$ & 2015 & $\begin{array}{l}\text { Arquivos Brasileiros } \\
\text { de Cardiologia }\end{array}$ & $\begin{array}{l}\text { Avaliar incidência e preditores de complicações e } \\
\text { óbito, assim como o desempenho de dois modelos de } \\
\text { estratificação de risco, em cirurgia vascular }\end{array}$ \\
\hline $\begin{array}{l}\text { Isquémia do cólon na cirurgia do } \\
\text { aneurisma da aorta abdominal }\end{array}$ & 2017 & $\begin{array}{c}\text { Sociedade } \\
\text { Portuguesa de } \\
\text { Angiologia e } \\
\text { Cirurgia Vascular }\end{array}$ & $\begin{array}{l}\text { O objetivo deste trabalho é analisar a sua frequência } \\
\text { nos doentes tratados por AAA e procurar identificar } \\
\text { fatores preditivos para a ocorrência de modo a permitir } \\
\text { diagnóstico precoce e intervenção terapêutica adequada } \\
\text { em tempo oportuno. }\end{array}$ \\
\hline $\begin{array}{l}\text { Outcomes of surgery for patientes } \\
\text { with Behcet's diese causing aortic } \\
\text { pseudoaneurysm: a shift from open } \\
\text { surgery to endovascular repair }\end{array}$ & 2016 & Clinics & $\begin{array}{l}\text { Investigar os resultados da doença de Behçet em } \\
\text { pacientes com pseudoaneurismas aórticos submetidos a } \\
\text { cirurgia aberta e correção endovascular aórtica }\end{array}$ \\
\hline $\begin{array}{l}\text { Qualidade de vida em pacientes } \\
\text { com doença arterial periférica }\end{array}$ & 2018 & $\begin{array}{l}\text { Jornal Vascular } \\
\text { Brasileiro }\end{array}$ & $\begin{array}{c}\text { Avaliar a qualidade de vida em pacientes com doença } \\
\text { arterial periférica internados no serviço de cirurgia } \\
\text { vascular em um hospital terciário beneficente }\end{array}$ \\
\hline $\begin{array}{l}\text { Reinforced aortic root } \\
\text { reconstruction for acute type A } \\
\text { aortic dissection involving the } \\
\text { aortic root }\end{array}$ & 2013 & $\begin{array}{l}\text { Brazilian Journal of } \\
\text { Cardiovascular } \\
\text { Surgery }\end{array}$ & $\begin{array}{l}\text { Existem debates sobre a abordagem ideal para } \\
\text { dissecção aguda de aorta tipo A (AAAD) envolvendo a } \\
\text { raiz da aorta. Descrevemos uma abordagem modificada } \\
\text { de reconstrução da raiz aórtica reforçada para o } \\
\text { tratamento de AAAD envolvendo a raiz da aorta. }\end{array}$ \\
\hline $\begin{array}{l}\text { Revisão dos outcomes cirúrgicos da } \\
\text { correção de fístulas aórticas: A } \\
\text { experiência de um serviço }\end{array}$ & 2020 & $\begin{array}{c}\text { Angiologia e } \\
\text { Cirurgia Vascular }\end{array}$ & $\begin{array}{c}\text { Rever os outcomes da intervenção cirúrgica em doentes } \\
\text { com fístulas com origem na aorta torácica descendente } \\
\text { e abdominal através da consulta dos processos clínicos } \\
\text { durante um período de } 16 \text { anos num departamento de } \\
\text { Cirurgia Vascular }\end{array}$ \\
\hline Síndrome pós-implante no EVAR & 2018 & $\begin{array}{c}\text { Angiologia e } \\
\text { Cirurgia Vascular }\end{array}$ & $\begin{array}{l}\text { Estimar a incidência de Síndrome pós-implante na } \\
\text { exclusão endovascular de aneurismas de aorta } \\
\text { abdominal (EVAR) na sua instituição, identificar } \\
\text { factores de risco e determinar a associação a eventos } \\
\text { clínicos }\end{array}$ \\
\hline $\begin{array}{l}\text { The evaluation of surgical } \\
\text { reconstruction for intermittent } \\
\text { claudication by the Walking } \\
\text { Impairment Questionnaire }\end{array}$ & 2010 & $\begin{array}{l}\text { Jornal Vascular } \\
\text { Brasileiro }\end{array}$ & $\begin{array}{l}\text { Avaliar a correlação entre o fluxo sanguíneo arterial } \\
\text { pós-operatório e a edição japonesa do Walking } \\
\text { Impairment Questionnaire }\end{array}$ \\
\hline $\begin{array}{l}\text { Tratamento cirúrgico do linfedema } \\
\text { primário de membro inferior } \\
\text { associado a malformação venosa: } \\
\text { Relato de caso e revisão de } \\
\text { literatura }\end{array}$ & 2020 & $\begin{array}{c}\text { Angiologia e } \\
\text { Cirurgia Vascular }\end{array}$ & $\begin{array}{l}\text { Revisar o processo clínico eletrônico de um caso } \\
\text { clínico grave de linfedema do membro inferior, além de } \\
\text { fazer uma revisão de literatura sobre o tema }\end{array}$ \\
\hline
\end{tabular}

Fonte: Mendes et al. (2021). 


\subsection{A qualidade de vida do indivíduo que sofre de doença arterial periférica}

Quando se trata da abordagem de um paciente com problemas vasculares, como a doença arterial periférica (DAP), é cada vez mais importante tratar da qualidade de vida (QV) dessa pessoa. Isso significa considerar o indivíduo em toda a sua totalidade, dando espaço para que suas condições biopsicossociais ganhem território na discussão médica (Aragão et al., 2018).

A realidade brasileira, em que doenças crônicas não transmissíveis (e entram nesse âmbito as patologias vasculares) são cada vez mais evidentes, estando relacionadas com mais de 70\% das mortes no país, explicita ainda mais a importância de se estudar como a QV afeta o paciente e como ela pode ser utilizada para potencializar estratégias de impacto positivo (Aragão et al., 2018).

O estudo publicado por Aragão JA et al. (2018) que buscou traçar uma avaliação da QV em pessoas com DAP que se encontravam em um hospital de Aracaju, demonstrou que homens com maior escolaridade tinham escores de QV melhores, algo que, em relação às diferenças entre os sexos, pode se dar por conta de diferenças biológicas, pelos novos papéis sociais e pela expectativa de vida crescente. Já quando se trata da escolaridade, ressalta-se que aquele que compreende melhor as questões envolvendo sua condição tende a aderir melhor ao tratamento (Aragão et al., 2018).

\subsection{Aplicação do Walking Impairment Questionnaire em pacientes com claudicação intermitente consequente de quadro de arteriosclerose obliterante}

O Walking Impairment Questionnaire (WIQ) é um questionário voltado para pacientes com claudicação intermitente devido a um quadro de arteriosclerose obliterante (ASO). Ele analisa aspectos como a dor, a distância de caminhada e a velocidade (Nakashima \& Yamaguchi, 2010).

A qualidade de vida $(\mathrm{QV})$ de um paciente que sofre de isquemia no membro é um fator importante quando se trata da evolução de seu quadro clínico. A forma curta do questionário é bastante utilizada e examina fatores físicos e mentais por meio de 36 perguntas. A área de abrangência dessas perguntas envolve 8 campos que vão desde a saúde mental até a função social e passando por fatores como a dor corporal (Nakashima \& Yamaguchi, 2010).

O WIQ se mostrou bastante útil em relação à avaliação pós-operatória por sua rapidez e sua especificidade. Levando em conta os custos, pode ser interessante adotar a realização do questionário antes mesmo de serem realizados exames de imagem (Nakashima \& Yamaguchi, 2010).

A utilidade do WIQ encontra apoio tanto na avaliação sintomática quanto na avaliação da deterioração da capacidade de caminhar depois de uma cirurgia vascular (Nakashima \& Yamaguchi, 2010).

\subsection{Resultados cirúrgicos para tratamento de pseudoaneurisma aórtico causado por doença de Behçet}

A doença de Behçet (DB) é uma vasculite sistêmica de etiologia ainda desconhecida e incidência relativamente rara, sendo comum particularmente na denominada "Rota da Seda", região que se estende desde o Mediterrâneo até o extremo Oriente (Neves, Moraes, \& Gonçalves, 2006). Ela é caracterizada por uma apresentação clínica diversa, podendo variar desde lesões cutâneas, com aparecimento de úlceras orais e genitais, até alterações vasculares, neurológicas e gastrointestinais (Ferrão, Almeida, Marinho, Vasconcelos, \& Correia, 2015).

De acordo com estudo realizado por Shen et al. (2016), as complicações vasculares dessa patologia, denominadas de vasculobehçet (VB), possuem especial atenção, devido à sua elevada mortalidade. Assim, os autores analisaram a melhor via para o tratamento de pseudoaneurisma aórtico proveniente de $\mathrm{VB}$, discutindo-se entre a cirurgia aberta e o reparo aórtico endovascular (REV). 
Os resultados dessa pesquisa confirmaram a segurança da REV para pacientes com pseudoaneurisma aórtico em pacientes com DB, especialmente nos casos de emergência. Não houve mortes após os 30 dias de cirurgia, além disso, ocorreram 7 recorrências em 5 pacientes (50\%), consistindo em 6 recorrências aórticas e 1 recidiva anastomótica femoral. Por fim, concluiu-se que a medicação imunossupressora foi essencial para adiar a ocorrência e desenvolvimento de aneurismas recorrentes. Dessa forma, Shen e colaboradores demonstraram que essa alternativa cirúrgica é segura e possibilita um bom prognóstico para o paciente, traduzindo em uma melhor qualidade de vida.

\subsection{Avaliação de complicações cardiovasculares e óbito em cirurgia vascular}

Smeili e Lotufo (2015) realizaram uma pesquisa com 141 pacientes, a fim de avaliar a incidência de complicações cardiovasculares, desfecho e óbito em até 30 dias após a cirurgia. Observaram-se complicações cardiovasculares em 28 $(19,9 \%)$ pacientes e evolução para óbito em $20(14,2 \%)$ do total de casos registrados. As principais complicações de origem cardiovascular foram infarto do miocárdio, insuficiência cardíaca descompensada, acidente vascular cerebral e arritmia. 25\% dos óbitos ocorridos na amostragem correspondem à causa cardiovascular. Esses dados demonstram uma taxa elevada de eventos cardiovasculares e óbitos totais evidenciados na literatura.

Ainda, em trabalho desenvolvido por Pereira, Oliveira, Carvalho e Bellen (2016), dividiu-se 200 pacientes em três grupos: grupo I, sem doença coronariana; grupo II, com doença coronariana assintomática; grupo III, com doença coronariana sintomática. Houveram complicações pós-operatórias em 46 pacientes (23\%), sendo 11 (5,5\%) cardíacas, das quais a mais frequente no estudo foi arritmia (exceto fibrilação atrial), ocorridas em cinco pacientes (três destes do grupo II). A mortalidade precoce total foi de nove $(4,5 \%)$ pacientes, que aconteceram dentro de 30 dias do período após a cirurgia. Uma importante conclusão dos autores foi a importância da realização de avaliação pré-operatória para a detecção de pacientes com doença coronariana assintomática de forma precoce, a fim de evitar-se possíveis complicações e proporcionar uma melhor recuperação.

\subsection{Avaliação da qualidade de vida em pacientes portadores de varizes de membros inferiores submetidos a tratamento cirúrgico}

Varizes ou doença das veias varicosas (Vv) é uma doença venosa crônica (DCV) caracterizada por alterações funcionais e estruturais das veias, as quais são mais comumente observadas em membros inferiores. A DCV é altamente prevalente no mundo e o surgimento de $\mathrm{Vv}$ está relacionado à diversos fatores de risco, como: sexo feminino, gravidez, obesidade, idade, componente genético e atividades laborais em que o indivíduo permanece muito tempo em posição ortostática. Atualmente, a cirurgia está entre as principais formas de tratamento utilizadas para cura (Lins et al., 2012).

No referido artigo, os autores optaram por utilizar um questionário autoaplicável, denominado VEINES-QOL / Sym, para análise da qualidade de vida $(\mathrm{QV})$ dos pacientes com Vv que foram submetidos ao tratamento cirúrgico - 92 indivíduos ao todo, de diversas faixas etárias. O VEINES-QOL / Sym aborda fatores como sinais e sintomas, alteração no desempenho de atividades diárias e impacto psicológico. Portanto, permite avaliar a eficácia e o impacto dessa medida terapêutica na vida dos pacientes participantes do estudo. A ferramenta é empregada no pré e no pós-operatório, gerando um escore de avaliação no qual a pontuação é diretamente proporcional à percepção de qualidade de vida do paciente. É essencial compreender que a avaliação da QV por meio dos questionários depende de uma interpretação subjetiva do paciente com relação ao seu processo de saúde-doença (Rocha et al., 2020).

Constatou-se que pacientes na faixa etária de 30 a 40 anos apresentaram maiores escores no questionário do pósoperatório, enquanto nas demais faixas etárias não houve melhora significativa da qualidade de vida. A conclusão obtida é uma 
hipótese que está relacionada aos fatores de risco relacionados à doença, como a idade e as comorbidades: o comprometimento funcional, social e psicológico enfrentado por pacientes mais idosos e com mais doenças pré-existentes, por exemplo, é frequentemente maior do que aquele enfrentado por indivíduos de faixas etárias mais baixas e sem outras enfermidades, seja no pré ou pós-operatório. Para além disso, não houve diferença na QV dos demais subgrupos do estudo após passarem pelo tratamento cirúrgico de varizes em membros inferiores. (Rocha et al., 2020).

Ainda nessa perspectiva, de acordo com um relato de caso feito por Faccini, Arendt, Pereira e Oliveira (2019), a técnica cirúrgica CHIVA (abreviação do francês, Cure Conservatrice et Hemodynamique de l'Insufficience Veineuse en Ambulatoire), proposta por Claude Franchesci, em 1988, para tratamento de insuficiência venosa crônica, a qual evita a destruição da veia safena e colaterais, obteve resultados clinicamente e esteticamente satisfatórios. De acordo com os autores, essa técnica possui um papel importante no tratamento de insuficiência venosa crônica pelo seu caráter conservador com relação à safena. Ademais, concluiu-se que a adoção do eco-Doppler como rotina da equipe cirúrgica pode prevenir problemas relacionados com o sítio operatório.

\subsection{Reconstrução reforçada de raiz aórtica para dissecção aórtica tipo A envolvendo a raiz aórtica}

A dissecção aórtica aguda (DAA) é uma condição clínica caracterizada pela separação das camadas da aorta - em geral, camadas íntima e média -, resultando em um lúmen falso. Ela é a síndrome aórtica aguda mais frequente, apresentando hipertensão arterial sistêmica como o principal fator de risco, e seu diagnóstico é dado a partir da associação de sintomas clínicos e exames complementares. Ademais, a DAA é classificada como uma emergência cirúrgica devido à sua associação com altas taxas de mortalidade. Isso se dá pela possibilidade de rompimento da aorta e subsequente hemorragia. Dessa forma, o objetivo primário da cirurgia de emergência é evitar essa ruptura e que o paciente venha a óbito. (Borrego, Ferreira, \& Pinto , 2016).

Nesse estudo, os autores, cirurgiões, trazem uma nova abordagem para o tratamento cirúrgico de reconstrução de dissecção aórtica aguda tipo A com acometimento da raiz aórtica e, com ele, suas análises peri e pós-operatórias da técnica cirúrgica empregada, complicações, taxas de mortalidade e de sobrevida. De acordo com eles, apesar de haver consenso em relação à necessidade de tratamento operatório rápido, não o há para a técnica que deve ser usada nesses casos (Han et al., 2013).

Sua avaliação sobre os fatores supracitados aponta para certa melhoria na qualidade de vida após a implementação da nova estratégia. Observou-se essa evolução pela ausência de dissecção aórtica recorrente, aneurisma ou pseudoaneurisma de aorta, as quais são complicações conhecidas e regularmente relatadas no pós-operatório de cirurgias de substituição supracomissural de aorta ascendente e da válvula aórtica - outras possíveis abordagens cirúrgicas. Além disso, as taxas de mortalidade hospitalar $(6,2 \%)$ e de acompanhamento $(6,8 \%)$, que ocorreu por um período de 2 a 12 anos, não se mostraram mais altas do que aquelas visualizadas em estudos já publicados relacionados às técnicas cirúrgicas previamente adotadas para esses casos. Contudo, há a limitação de ser um estudo retrospectivo sem outro grupo para comparação (Han et al., 2013).

\subsection{Isquemia do cólon na cirurgia do aneurisma da aorta abdominal e Hérnias incisionais no pós-operatório de correção de aneurisma de aorta abdominal}

O aneurisma de aorta abdominal (AAA) é uma doença vascular que apresenta gradativo aumento de prevalência, estando relacionada a fatores de risco como idade e tabagismo. Sua evolução pode ser assintomática até a rotura da aorta, complicação de maior importância, dificultando o diagnóstico (Barros et al., 2005). Nos Estados Unidos e na Europa, o AAA é 
um quadro muitas vezes silencioso que representa a $10^{\mathrm{a}}$ maior causa de morte evitável. Por essa razão, o enfoque no rastreio é essencial para a redução da mortalidade e das complicações pós-operatórias (Moutinho et al., 2017).

Dentre as complicações pós-operatórias mais comuns da cirurgia de tratamento de AAA, está a isquemia de cólon (IC), também denominada de isquemia mesentérica. Os autores do estudo analisaram a prevalência da IC como complicação pós-operatória e os desfechos dos pacientes que a apresentam, comparando o grupo submetido à cirurgia eletiva com o submetido à cirurgia de emergência e urgência. A taxa de mortalidade dos pacientes, os quais apresentaram rotura, que fizeram a cirurgia e desenvolveram IC foi de $35,7 \%$ ao $30^{\circ}$ dia de acompanhamento pós-operatório, enquanto a taxa dos pacientes que não apresentaram rotura foi de 5\%, no mesmo período. $40 \%$ dos pacientes analisados apresentaram IC como complicação. Observa-se, pois, a diferença estatisticamente grande entre os grupos analisados (Moutinho et al., 2017).

Outrossim, foi evidenciada a importância do rastreio e do diagnóstico precoce da IC em pacientes submetidos à cirurgia vascular de reparação da doença aneurismática aórtica como a melhor forma, até então, de evitar a morbimortalidade associada e oferecer melhor qualidade de vida ao paciente (Moutinho et al., 2017).

Do mesmo modo, hérnias incisionais (HI) também constituem uma complicação comum - e principal indicação de reoperação - após cirurgia de correção de AAA. Seu diagnóstico é confirmado, em geral, a partir de exames de imagem, como ultrassonografia ou tomografia computadorizada de abdômen, estando presentes em 10\% - 37\% dos pacientes no pósoperatório (Menezes \& Guillaumon, 2012).

É descrito que o processo de formação da hérnia é multifatorial, o qual também apresenta fator genético influenciador, como na Síndrome de Marfan e Ehlers-Danlos. Apesar do reconhecimento das doenças anteriormente citadas e de fatores externos, como tabagismo, na qualidade de agentes relacionados ao maior aparecimento de HI, os autores reforçam a importância de empregar a técnica cirúrgica correta para o fechamento da aponeurose como a forma mais favorável de evitar a nova operação desses pacientes, tornando o pós-operatório menos complicado e, assim, corroborar para melhor qualidade de vida. (Menezes \& Guillaumon, 2012).

\subsection{Ocorrência de Infarto Agudo do Miocárdio em indivíduos com isquemia crítica passados por cirurgia de revascularização de membros inferiores}

Pessoas portadoras de isquemia crítica dos membros inferiores que se constituem como candidatas adequadas para uma cirurgia de revascularização desses membros devem ser particularmente acompanhadas devido ao risco de um infarto agudo do miocárdio (IAM) acontecer. Pacientes com esse quadro são mais suscetíveis a um IAM por conta do estresse cirúrgico com a produção aumentada de catecolaminas. Não por acaso, essa complicação desponta como importante causa de morte no perioperatório da cirurgia (Lins et al., 2013).

Uma boa avaliação cardiovascular capaz de atestar a presença de uma isquemia é essencial em pacientes com DAOP. Nas pessoas com DAOP, também é mais comum se atestar casos de doença arterial coronariana (DAC). Em ambos os casos, as chances de o paciente necessitar de cirurgia são altas devido, principalmente, à aterosclerose (Lins et al., 2013).

\subsection{Presença de linfedema primário de membro inferior associado a malformação venosa e seu tratamento}

A presença de edema intersticial decorrente de uma dificuldade na drenagem da linfa caracteriza o linfedema dos membros inferiores, condição que pode exercer um impacto negativo relevante na $\mathrm{QV}$ de alguém afetado. $\mathrm{O}$ tratamento cirúrgico só costuma ser indicado em casos mais graves, mais comum é que se lance mão de terapêutica compressiva e de massagem de drenagem linfática. As primeiras manifestações desse problema são edema nos membros inferiores com sinal de Godet positivo. Com o tempo, a pele e o tecido subcutâneo se espessa, associando-se a um processo de fibrose e 
hiperqueratose. Pode, ainda, haver a formação de vesículas cutâneas com linforragia. Já a dor costuma vir acompanhada de casos de infecção (Mendes et al., 2020).

O linfedema primário (LP) ocorre fruto de anomalias do próprio sistema linfático (ao contrário do secundário, fruto de um fator externo). A International Society of Lymphology divide o linfedema em 3 graus: o I, em que o edema é mole e sem fibrose; o II, com edema irredutível e com fibrose de moderada a grave; o III, em que o edema é irreversível e com fibrose e esclerose de pele e de tecido subcutâneo. O diagnóstico e pauta na história e no exame clínico, com exames complementares sendo utilizados na eliminação de diagnósticos diferenciais e ajudando a delimitar qual o padrão que a enfermidade apresenta (Mendes et al., 2020).

O linfedema não oferece perspectiva de cura e o tratamento não invasivo tem sido o de escolha em grande parte dos casos. Já o tratamento cirúrgico se destina, em especial, a casos em que algo mais conservador foi tentado, mas não surtiu os efeitos esperados. Nesse último caso, é feita uma cirurgia reconstrutiva, em que se visa a melhoria da drenagem da linfa, seguida de uma cirurgia redutora, que objetiva a correção do que o linfedema deformou (Mendes et al., 2020).

No caso clínico explicitado por Mendes D et al. (2020), o linfedema do paciente associou-se a uma malformação venosa, algo raro. O tratamento de escolha se deu por meio da recessão extensa da pele e do tecido celular subcutâneo da coxa. Com isso, alcançou-se o resultado esperado de redução das deformidades, culminando numa melhora quanto aos problemas da linforragia e da dificuldade de deambulação no pós-operatório, o que proporcionou melhoria na qualidade de vida do paciente. (Mendes et al., 2020).

\subsection{Fístulas na aorta e resultados do tratamento cirúrgico}

As fístulas que se originam na aorta ainda estão associadas a uma alta mortalidade. Elas podem ser primárias ou secundárias, sendo aquelas as que representam uma comunicação espontânea entre a aorta e alguma estrutura adjacente, vaso ou órgão, e bem mais raras. Já estas se referem a uma cirurgia aórtica anterior. A localização mais comum para o aparecimento de fístulas aórticas (FA) é a terceira porção duodenal e única opção terapêutica para o indivíduo afetado é o procedimento cirúrgico (Melo et al., 2020).

O estudo de Melo et al. (2020) encontrou uma taxa de mortalidade intra-hospitalar de $50 \%$ e de amputação maior de membro inferior de $25 \%$. A taxa de mortalidade observada foi maior do que a encontrada num estudo de revisão da literatura, mas ressaltou-se que o estudo citado considerava pacientes tratados por via endovascular, apresentando uma tendência maior de apresentar resultados positivos. Já o de Melo et al. (2020) não considerou pacientes tratados por essa via em seus estudos.

\subsection{Ocorrência de síndrome pós-implante na exclusão endovascular de aneurismas de aorta abdominal}

A exclusão endovascular de aneurismas de aorta abdominal (EVAR) consiste numa operação pouco invasiva que, entretanto, está relacionada a uma síndrome pós-implante (SPI), que se refere a reações inflamatórias no pós-operatório, reações estas que impactam de maneira desfavorável a vida do indivíduo que passou por uma operação de EVAR. Para se definir a existência de uma SPI, é preciso que se haja resposta positiva de dois critérios: leucócitos acima de 12000/ml e temperatura acima de $38^{\circ}$ ou haver critérios de temperatura acima de $38^{\circ}$ e elevação da PCR (Ferreira et al., 2018).

No estudo de Ferreira RS et al. (2018), foi encontrada uma incidência de 19\% de SPI após a realização de EVAR. O estudo traz o tabagismo como um fator de risco e o uso de estatina como fator de proteção. Nas pessoas que apresentaram essa síndrome, o tempo de internação foi, em média, acrescido de dois dias, além de ensaios analisados atestarem uma influência negativa na sobrevida e no aparecimento de eventos adversos cardiovasculares nos pacientes, tudo isso impactando diretamente na qualidade de vida dessas pessoas. Nesse sentido, o uso profilático de corticosteróide no pré-operatório aparenta 
reduzir a incidência da SPI (Ferreira et al., 2018).

\section{Conclusão}

Pacientes acometidos por doenças vasculares necessitam de um cuidado especial no intuito de evitar complicações no pós-operatório e garantir uma boa qualidade de vida. Observou-se que essa atenção deve estar voltada não apenas para o conjunto operatório que inclui ações pré, peri e pós-operatório, mas também fatores de risco e aspectos biopsicossociais cujo paciente está inserido. Sabe-se que a qualidade de vida no pós-operatório está intimamente relacionada à gravidade da doença, podendo o procedimento ser satisfatório ou não. Nesse âmbito, avaliou-se que o rastreio das principais complicações, associado à melhoria das técnicas cirúrgicas e à aplicação do melhor procedimento, tem impacto significativo na redução da morbimortalidade e melhor recuperação dos pacientes. Ainda, a análise dos fatores de risco, como idade, tabagismo e comorbidades associadas, permite definir a melhor forma de tratamento e suas possíveis repercussões orgânicas. Por fim, a avaliação da realidade que o paciente está inserido tem importância considerável, uma vez que é possível compreender seu entendimento com o processo de adoecimento e, consequentemente, potencializar os efeitos positivos do seu tratamento. Dessa maneira, têm-se que a qualidade de vida dos pacientes no pós-operatório de cirurgia vascular tem íntima relação com fatores diversos, desde a técnica em si a fatores subjetivos de cuidado.

\section{Referências}

Aragão, J. A., Santos, R. M., Neves, O. M. G., Aragão, I. C. S., Aragão, F. M. S., Mota, M. I. A., Bastos, R. de S. M., \& Reis, F. P. (2018). Qualidade de vida em pacientes com doença arterial periférica. Jornal Vascular Brasileiro, 17 (2), 117-121. https://doi.org/10.1590/1677-5449.009017

Barros, F. S., Pontes, S. M., Taylor, M. A. S. A., Roelke, L. H., Sandri, J. L., Jacques, C. de M., Zandonade, E., Nefal, D. P., De Vita, J. A., Borges, C. A., Sandri, G de A., \& Moreira, I. G. (2005). Rastreamento do aneurisma da aorta abdominal na população da cidade de Vitória (ES). Jornal Vascular Brasileiro, 4 (1), 59-65. JVasc-05-1-b-pdf.p65 (jvascbras.org)

Borrego, A., Ferreira, P. C., \& Pinto, F. J. (2017). Acute type A aortic dissection in a patient with paraganglioma. Portuguese Journal of Cardiology, 36 (10), 777. 10.1016/j.repc.2016.08.013

Faccini, F. P., Arendt, A. L., Pereira, R. Q., \& Oliveira, A. R. (2019). CHIVA to spare the small and great saphenous veins after wrong-site surgery on a normal saphenous vein: a case report. Jornal Vascular Brasileiro, 18, e20180077. https://doi.org/10.1590/1677-5449.007718

Ferrão, C., Almeida, I., Marinho, A., Vasconcelos, C., \& Correia, J. A. (2015). A nossa regra de ouro na doença de Behçet: Tratar a manifestação clínica. Arquivos de Medicina, 29 (3), 75-79. http://www.scielo.mec.pt/scielo.php?script=sci_arttext\&pid=S0871-34132015000300003\&lng=pt\&tlng=pt

Ferraz, S. M., Moreira, J. P., Mendes, L. C., Amaral, T. M., Andrade, A. R., Santos, A. R., \& Abelha, F. J. (2018). Avaliação da qualidade da recuperação e do estado de saúde no pós-operatório de cirurgias eletivas. Revista Brasileira de Anestesiologia, 68 (6), 577-583. https://doi.org/10.1016/j.bjane.2018.06.002

Ferreira, R. S., Gonçalves, F. B., Abreu, R., Camacho, N., Catarino, J., Correia, R., \& Ferreira, M. E. (2018). Síndrome pós-implante no EVAR. Angiologia e Cirurgia Vascular, 14 (3), 176-181. http://www.scielo.mec.pt/scielo.php?script=sci_arttext\&pid=S1646-706X2018000300003\&lng=pt\&tlng=pt

Giaretta, D. G., Vigueras, E. S. R., Ruschel P. P., \& Souza C (2018). Qualidade de vida e resiliência em pacientes pós-operatório de cirurgia cardíaca. Revista de Atenção à Saúde, 16 (58), 5-11. 10.13037/ras.vol16n58.5399

Han, Q., Song, Z., Han, L., Lu, F., Lang, X., \& Xu, Z. (2013). Reinforced aortic root reconstruction for acute type A aortic dissection involving the aortic root. Brazilian Journal of Cardiovascular Surgery, 28 (2), 190-199. https://dx.doi.org/10.5935/1678-9741.20130028

Lins, E. M., Barros, J. W., Appolonio, F., Anacleto, E., \& Lima, E. C. (2013). Acute myocardial infarctation in patients with critical ischemia underwent lower limb revascularization. Jornal Vascular Brasileiro, 12 (4), 284-288. https://doi.org/10.1590/jvb.2013.059

Lins, E. M., Barros, J. W., Appolônio, F., Lima, E. C., Barbosa Junior, M., \& Anacleto, E. (2012). Perfil epidemiológico de pacientes submetidos a tratamento cirúrgico de varizes de membros inferiores. Jornal Vascular Brasileiro, 11 (4), 301-304. https://dx.doi.org/10.1590/S1677-54492012000400008

Melo, M. B. de, Constâncio, V., Silva, J., Lima, P., Moreira, M., Canhoto, C., Antunes, L., \& Gonçalves, Ó. (2020). Revisão dos outcomes cirúrgicos da correção de fístulas aórticas: A experiência de um serviço. Angiologia e Cirurgia Vascular, 16 (1), 17-23. http://www.scielo.mec.pt/scielo.php?script=sci_arttext\&pid=S1646-706X2020000100004\&lng=pt\&tlng=pt 
Research, Society and Development, v. 10, n. 2, e20110212139, 2021

(CC BY 4.0) | ISSN 2525-3409 | DOI: http://dx.doi.org/10.33448/rsd-v10i2.12139

Mendes, D., Machado, R., Mesquita, A., Antunes, I., Veiga, C., Veterano, C., Rocha, H., Castro, J., Pinelo, A., \& Almeida, R. de. (2020). Tratamento cirúrgico do linfedema primário de membro inferior associado a malformação venosa: Relato de caso e revisão de literatura. Angiologia e Cirurgia Vascular, 16 (1), 53-57. http://www.scielo.mec.pt/scielo.php?script=sci_arttext\&pid=S1646-706X2020000100011\&lng=pt\&tlng=pt

Menezes, F. H., \& Guillaumon, A. T. (2012). Hérnias incisionais no pós-operatório de correção de aneurisma de aorta abdominal. Jornal Vascular Brasileiro, 11 (3), 182-186. https://doi.org/10.1590/S1677-54492012000300003

Moutinho, M., Fernandes, R., Silvestre, L., Evangelista, A., Sobrinho, G., Ministro, A., Pedro, L. M., \& Fernandes, J. F. (2017). Isquémia do cólon na cirurgia do aneurisma da aorta abdominal. Sociedade Portuguesa de Angiologia e Cirurgia Vascular, 13 (3), 10-17. http://www.scielo.mec.pt/scielo.php?script=sci_arttext\&pid=S1646-706X2017000300004\&lng=pt\&tlng=pt

Nakashima, M., \& Yamaguchi, K. (2010). The evaluation of surgical reconstruction for intermittent claudication by the Walking Impairment Questionnaire Jornal Vascular Brasileiro, 9 (4), 215-219. https://doi.org/10.1590/S1677-54492010000400002

Neves, F. de S., Moraes, J. C. B. de, \& Gonçalves, C. R. (2006). Síndrome de Behçet: À procura de evidências. Revista Brasileira de Reumatologia, 46 (1), 21-29. https://doi.org/10.1590/S0482-50042006000700005

Pereira, K. S. M., Oliveira, J. C. P., Carvalho, F. C., Bellen, B. V. (2016). Cardiac complications in vascular surgery. Jornal Vascular Brasileiro, 15 (1), $16-$ 20. https://doi.org/10.1590/1677-5449.003515

Sociedade Brasileira de Angiologia e de Cirurgia Vascular. (2015). Insuficiência venosa crônica diagnóstico e tratamento.

Rocha, F. A., Lins, E. M., De Almeida, C. C., Dias Junior, R. C., Da Silva, P. A. L., Gameleira, C. A., Falcão, M. G de M. G., \& Barros, J. W. dos S. (2020). Avaliação da qualidade de vida em pacientes portadores de varizes de membros inferiores submetidos a tratamento cirúrgico. Jornal Vascular Brasileiro, 19, e20190108. https://doi.org/10.1590/1677-5449.190108

Shen, C., Li, W., Zhang, Y., Li, Q., Jiao, Y., Zhang, T., \& Zhang, X. (2016). Outcomes of surgery for patientes with Behcet's diese causing aortic pseudoaneurysm: a shift from open surgery to endovascular repair. Clinics, 71 (6), 302-310. https://doi.org/10.6061/clinics/2016(06)03

Smeili, L. A. A., Lotufo, P. A. (2015). Incidência e preditores de complicações cardiovasculares e óbito após cirurgia vascular arterial. Arquivos Brasileiros de Cardiologia, 105 (5), 510-518. 10.5935/abc.20150113 